REGARDS

SUR L'ECONOMIE ALLEMANDE

BULLETIN ECONOMIQUE DU CRAC
Regards sur l'économie allemande

Bulletin économique du CIRAC

$91 \mid 2009$

Varia

\title{
Un marché de l'emploi en mutation
}

\section{Eugen Spitznagel}

Traducteur : Isabelle Bourgeois et Solène Hazouard

\section{OpenEdition}

Journals

Édition électronique

URL : http://journals.openedition.org/rea/3671

DOI : 10.4000/rea.3671

ISBN : 978-2-8218-0878-2

ISSN : 1965-0787

Éditeur

CIRAC

Édition imprimée

Date de publication : 1 mai 2009

Pagination : 5-16

ISSN : 1156-8992

\section{Référence électronique}

Eugen Spitznagel, «Un marché de l'emploi en mutation », Regards sur l'économie allemande [En ligne],

91 I mai 2009, mis en ligne le 01 mai 2011, consulté le 02 mai 2019. URL : http://

journals.openedition.org/rea/3671; DOI : 10.4000/rea.3671 


\section{Un marché de l'emploi en mutation}

\section{Eugen Spitznagel}

En Allemagne, la crise financière globale a brutalement mis fin à une période relativement longue de forte croissance économique. Elle a plongé le pays dans une récession dont nul ne peut encore estimer aujourd'hui ni la profondeur, ni la durée. Mais d'ores et déjà, elle se révèle plus forte que les crises conjoncturelles qu'a connues l'Allemagne au cours des années 1966-1967, 1974-1975, 1993 et 2003 : on estime généralement qu'en 2009, le PIB connaîtra un recul de $6 \%$. Les réformes structurelles de ces dernières années, les récentes mesures de soutien à la conjoncture, ainsi que les mutations démographiques laissent cependant présager aujourd'hui d'une meilleure résistance du marché de l'emploi que lors des crises passées. Du moins au début de cette phase de récession, celui-ci s'est-il montré particulièrement robuste.

\section{Un marché de l'emploi en dents de scie depuis les années 1970}

L'Allemagne a connu le plein-emploi pour la dernière fois au début des années 1970. Ensuite, le chômage n'a cessé d'augmenter en tendance, par paliers successifs. Certes, au cours des années 1980, le nombre des actifs occupés s'est accru de près de 4 millions de personnes, ce qui a porté le total des actifs occupés à 30,2 millions de personnes en 1991, soit près de 3,5 millions de plus qu'au début des années 1970. Mais durant la même période, l'offre d'emploi s'est elle aussi accrue car le marché de l'emploi s'était étendu sous l'effet de l'arrivée massive sur le territoire ouest-allemand de réfugiés de l'ex-RDA, d'immigrants de souche allemande et d'immigrants étrangers. Au total, le marché du travail a alors intégré un nouveau potentiel de main-d'œuvre de sept millions de personnes. Le volume de création d'emplois étant insuffisant en comparaison, le nombre de chômeurs augmenta, atteignant 1,6 million de personnes en 1991 en Allemagne de l'ouest. Parallèlement augmentait le chômage caché, la "réserve latente ", c'est-à-dire la catégorie des personnes qui aimeraient travailler mais ne sont pas inscrites au chômage ; elle comptait alors 800000 personnes.

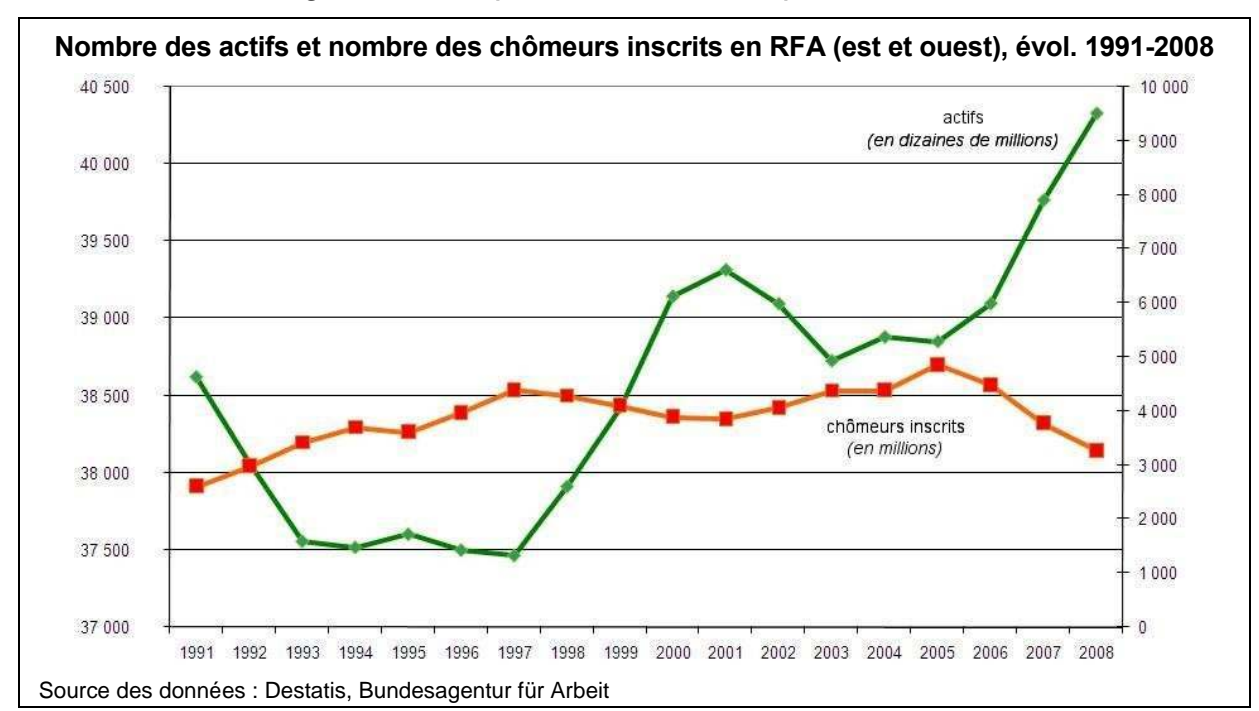

Avant I'Unité, 1,6 million de chômeurs à l'ouest 
Années 1990, transition à l'est et crise économique gonflent le nombre de chômeurs
1998-2001 : une embellie de courte durée
La réunification a d'abord déclenché un boom économique, dans la seule Allemagne de l'ouest toutefois. Dans les nouveaux Länder, la transition économique a entraîné dans les années suivant l'Unité (1990) la perte d'un tiers des emplois par rapport à l'époque de la RDA. S'est ajoutée à cela la récession mondiale, qui a atteint l'Allemagne en 1993. Après une brève reprise économique en 1994, l'Allemagne a traversé une longue période de faible croissance. Au total, entre 1991 et 1997, le nombre d'actifs occupés s'est ainsi réduit de 1,16 million. Plus précisément, le nombre de demandeurs d'emploi s'est accru de 890 000, et le nombre de chômeurs indemnisés de près de 1,78 million de personnes pour atteindre 4,4 millions au total. Par ailleurs, le nombre de demandeurs d'emploi non inscrits a augmenté de 490000 , portant l'effectif de la réserve latente à plus de 2 millions de personnes.

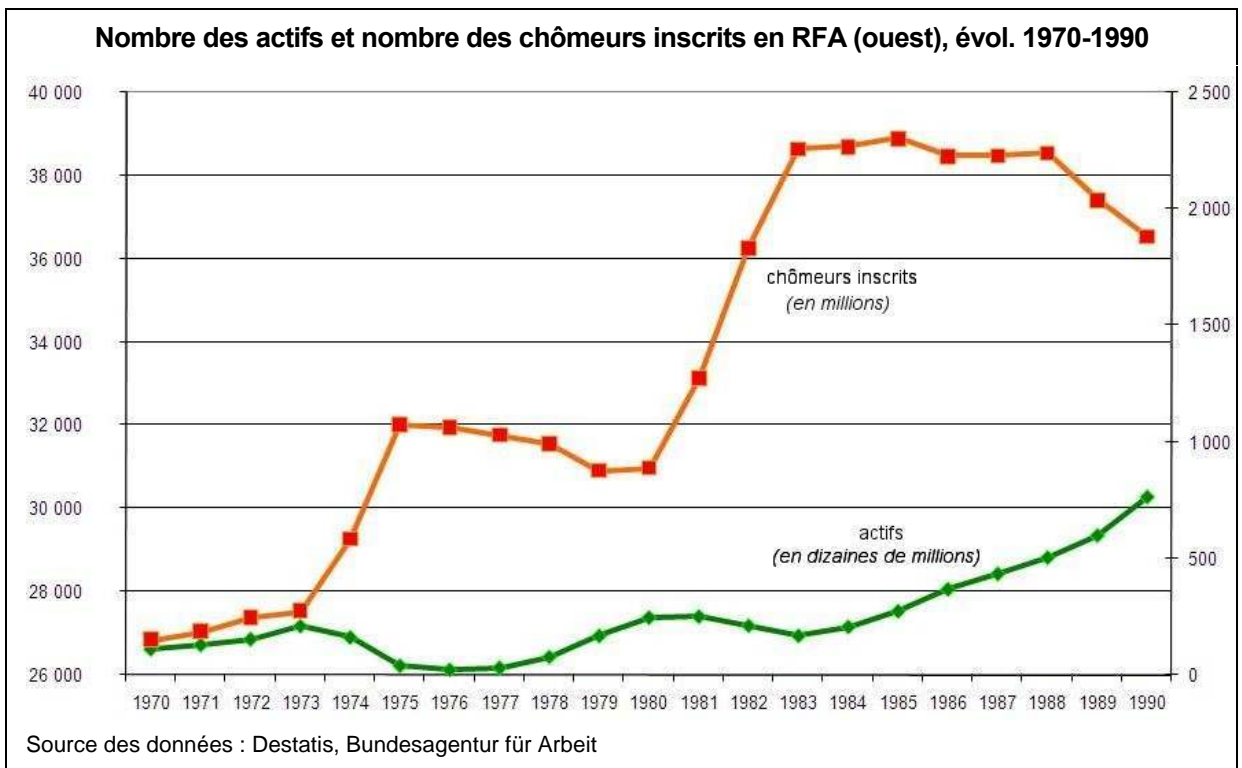

La reprise conjoncturelle des années 1998-2001 a certes généré des emplois, et le nombre de chômeurs reculait à 3,89 millions de personnes. Mais l'embellie n'a été que passagère, le ralentissement conjoncturel suivant la forte croissance de 1999/2000 les détruisant à nouveau. Et sous l'effet d'une demande d'emploi en progression, le nombre de chômeurs est remonté à 4,06 millions en 2002.

Evol. 1961-2008 du PIB réel de l'Allemagne (évol. par rapport à l’année précédente, en \%)

2003-05 : les « réformes Hartz » ont permis d'intégrer le chômage caché dans les statistiques

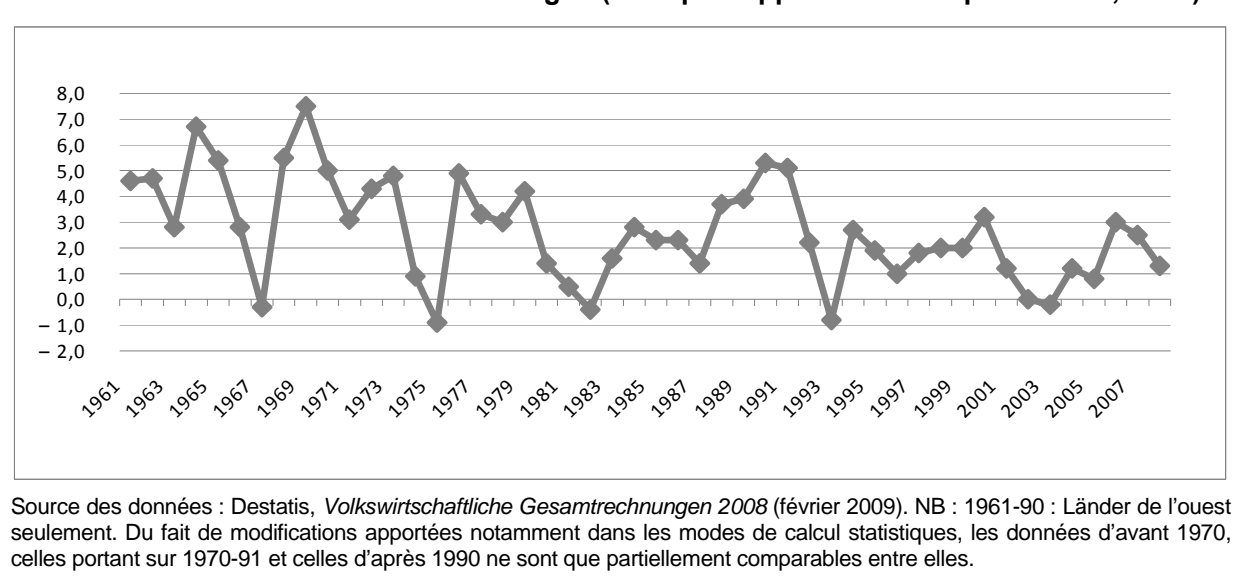

Les années 2003-2005 sont marquées par l'entrée en vigueur progressive des dispositions des « réformes Hartz ». Dans un premier temps, elles n'ont guère eu d'impact sur le marché de l'emploi : la croissance restant faible, le nombre des 
actifs occupés a continué de reculer tandis que celui des chômeurs progressait pour s'établir à près de 4,7 millions en 2005.

\begin{abstract}
Les quatre Lois pour des services modernes du marché de l'emploi (Gesetze für moderne Dienstleistungen am Arbeitsmarkt), plus connues sous le nom de " lois Hartz ", adoptées dans le cadre de la politique de réforme structurelle (Agenda 2010) du second gouvernement Schröder, sont entrées en vigueur progressivement entre janvier 2003 et janvier 2005. Les trois premières visent à accroître l'efficience du système d'assurance chômage (financé par les cotisations sociales) ; la quatrième, à rétablir l'équilibre entre prestations d'assurance (allocations chômage) et prestations d'assistance (revenu minimum), ces dernières étant financées par l'impôt. Le pivot de cette nouvelle approche globale est de mieux concilier responsabilité individuelle et protection collective.

La loi « Hartz I » (2003) durcit notamment le régime d'indemnisation des chômeurs, exigeant des bénéficiaires une plus grande mobilité ou l'acceptation d'un emploi au profil de qualification différent, soit encore une rémunération inférieure. La loi « Hartz II » (2003) élargit les dispositions relatives aux petits boulots (par ex. création des « minijobs »). La loi « Hartz III » (2004) restructure l'Office de Nuremberg, rebaptisé Agence fédérale pour l'emploi (Bundesagentur für Arbeit, BA) et rationalise le régime des prestations de l'assurance chômage (elle ramène notamment à 12 mois pour les moins de 50 ans la durée de versement des allocations). Les allocations chômage sont dénommées depuis : "Arbeitslosengeld I 》 (ALG I).

La loi « Hartz IV » (2005) modifie les deux régimes antérieurs d'assistance. Elle supprime l'ancienne aide aux chômeurs en fin de droits et la remplace par un " minimum garanti pour les personnes en quête d'emploi » (Grundsicherung für Arbeitssuchende), dénommé “Arbeitslosengeld II 》 (ALG II). Ce revenu minimum d'insertion comprend deux volets: des mesures de (re)qualification et d'insertion, et un revenu de substitution de base dont le montant, désormais forfaitaire (et non plus proportionnel à l'ancien salaire), est équivalent à celui de l'aide sociale (Sozialhilfe). Celle-ci, outil de lutte contre l'exclusion qui s'adresse aux seules personnes incapables de travailler et donc en mal d'insertion sociale, est maintenue. L'ALG // est destiné à l'opposé aux personnes disposant d'une capacité de travail pleine et entière (au moins 3 heures/jour) et se trouvant en quête active d'emploi. Contrairement au régime antérieur, aide sociale et $A L G$ // ne sont plus cumulables. Et en distinguant ces deux types de revenus de transfert (financés par l'impôt), la loi « Hartz IV » redéfinit la notion de demandeur d'emploi, la mettant en conformité avec les pratiques en vigueur dans l'UE.
\end{abstract}

Cette forte hausse a également pour origine l'entrée en vigueur en janvier 2005 du dernier volet des « réformes Hartz », la loi « Hartz IV », qui modifie en profondeur l'architecture du système d'indemnisation chômage. Depuis 2005, tous les anciens bénéficiaires de l'aide sociale doivent, dès lors qu'ils bénéficient d'une capacité de travail pleine et entière, s'inscrire comme demandeurs d'emploi s'ils veulent bénéficier du nouveau RMI, l'ALG II. Cette mesure a en quelque sorte 'officialisé' le chômage caché qui a ainsi pu être pris en compte par les statistiques.

\section{Depuis 2006, la structure du marché de l'emploi a subi de profondes mutations sous l'effet de la flexibilisation}

La période 2006-2008 a été marquée par une forte croissance économique. Parallèlement, les réformes du marché de l'emploi entrées en vigueur peu de temps auparavant commencent à déployer leurs premiers effets. Si on compare ces années avec la période de croissance 1999-2001, on observe des différences significatives qui révèlent les effets des réformes du marché de l'emploi.

En effet, si l'économie a connu durant ces périodes triennales une croissance comparable en données réelles, respectivement de $6,9 \%$ et de $6,6 \%$, l'évolution du marché de l'emploi diffère fortement d'une période à l'autre, comme en témoignent les données relatives à l'offre et la demande d'emploi, au taux de chômage, mais aussi à la politique de l'emploi dans son ensemble comme à la structure des diverses formes d'emploi (Bach/Spitznagel, 2007).

Entre 1999 et 2001, le nombre d'actifs occupés a augmenté de 1,41 million $(+3,7 \%)$; durant les années 2006-2008, la hausse n'a été que légèrement supérieure $(+3,8 \%$ ou 1,48 million de personnes de plus), alors que la croissance était elle aussi légèrement supérieure (tableau 1). On pourrait en conclure, un peu hâtivement, que la dernière période de croissance a été aussi créatrice d'emplois que celle des années 1999-2001. Une analyse plus poussée montre cependant qu'il y a dix ans, ce sont exclusivement des emplois à temps partiel qui furent créés, qu'il s'agisse de petits boulots de type « mini-jobs » (778 000) ou d'emplois à temps partiel 'classiques', c'est-à-dire soumis à cotisations sociales $(740000)$. Dans le même temps, le nombre d'emplois à temps plein diminuait (-231 000).
2006-08 : les réformes Hartz déploient leurs effets

1999-2001 : création d'emplois à temps partiel exclusivement, surtout des 'petits boulots' 
Tableau 1:

1999-2001 et 2006-2008 : croissance économique et variations du marché de l'emploi

\begin{tabular}{|c|c|c|c|c|}
\hline & \multicolumn{4}{|c|}{ Variations } \\
\hline & \multicolumn{2}{|c|}{$1999-2001$} & \multicolumn{2}{|c|}{ 2006-2008 } \\
\hline & $\%$ & 1000 & $\%$ & 1000 \\
\hline \multicolumn{5}{|l|}{ Offre d'emploi } \\
\hline $\begin{array}{l}\text { Potentiel d'actifs } \\
\text { Solde démographique } \\
\text { Comportement d'activité } \\
\text { Solde migratoire }\end{array}$ & $+1,1$ & $\begin{array}{r}+496 \\
-709 \\
+960 \\
+245\end{array}$ & $-0,4$ & $\begin{array}{r}-172 \\
-345 \\
+164 \\
+9\end{array}$ \\
\hline \multicolumn{5}{|l|}{ Demande d'emploi } \\
\hline $\begin{array}{l}\text { PIB, en valeur corrigée de l'inflation } \\
\text { Productivité horaire }\end{array}$ & $\begin{array}{l}+6,6 \\
+5,9\end{array}$ & & $\begin{array}{l}+6,8 \\
+3,1\end{array}$ & \\
\hline $\begin{array}{l}\text { Heures travaillées } \\
\text { Durée de travail effective }\end{array}$ & $\begin{array}{r}+6,0 \\
-3,0\end{array}$ & & $\begin{array}{r}+3,7 \\
-0,1\end{array}$ & \\
\hline $\begin{array}{l}\text { Actifs occupés } \\
\text { Indépendants et travailleurs familiaux } \\
\text { Salariés } \\
\text { - temps plein } \\
\text { - temps partiel } \\
\text { Temps partiel 'classique' } \\
\text { Petits boulots (estimation IAB) } \\
\text { Salariés sur le « } 1^{\mathrm{er}} \text { marché de l'emploi » (secteur marchand) } \\
\text { Salariés sur le « } 2^{\mathrm{e}} \text { marché de l'emploi » (secteur non marchand) } \\
\text { Actifs occupant un emploi soumis à cotisations sociales } \\
\text { Emploi intérimaire }\end{array}$ & $\begin{array}{r}\mathbf{+ 3 , 7} \\
+3,1 \\
+3,8 \\
-0,9 \\
+18,0 \\
+17,0 \\
+19,1 \\
+3,6 \\
+17,0 \\
+2,4 \\
+38,6\end{array}$ & $\begin{array}{r}+1405 \\
+118 \\
+1287 \\
-231 \\
+1518 \\
+740 \\
+778 \\
+1223 \\
+64 \\
+664 \\
+95\end{array}$ & $\begin{array}{r}+3,8 \\
+2,4 \\
+4,0 \\
+2,4 \\
+7,3 \\
+12,9 \\
+2,0 \\
+4,2 \\
-9,89 \\
+4,8 \\
+73,0\end{array}$ & $\begin{array}{r}+1479 \\
+105 \\
+1374 \\
+550 \\
+824 \\
+710 \\
+113 \\
+1415 \\
-41 \\
+1270 \\
+324\end{array}$ \\
\hline \multicolumn{5}{|l|}{ Bilan du marché de l'emploi } \\
\hline $\begin{array}{l}\text { Chômeurs inscrits } \\
\text { Dont chômeurs de longue durée } \\
\text { Réserve latente stricto sensu *) } \\
\text { Réserve latente bénéficiant de prog. de formation profes., etc. }\end{array}$ & & $\begin{array}{r}-428 \\
-245 \\
-484 \\
+37\end{array}$ & & $\begin{array}{r}-1593 \\
-500 \\
-228 \\
-14\end{array}$ \\
\hline Total sous-emploi & & -875 & & -1835 \\
\hline
\end{tabular}

Sources des données : Destatis, Agence fédérale pour l'emploi, calculs de l'Institut für Arbeitsmarkt und Berufsforschung (FG AZ). *) Réserve latente stricto sensu = personnes qui ne sont pas inscrites au chômage ni bénéficiaires des programmes de formation mais qui aimeraient travailler.

Tableau 2 :

2000-2007 : croissance économique et évolution de la structure de l'emploi

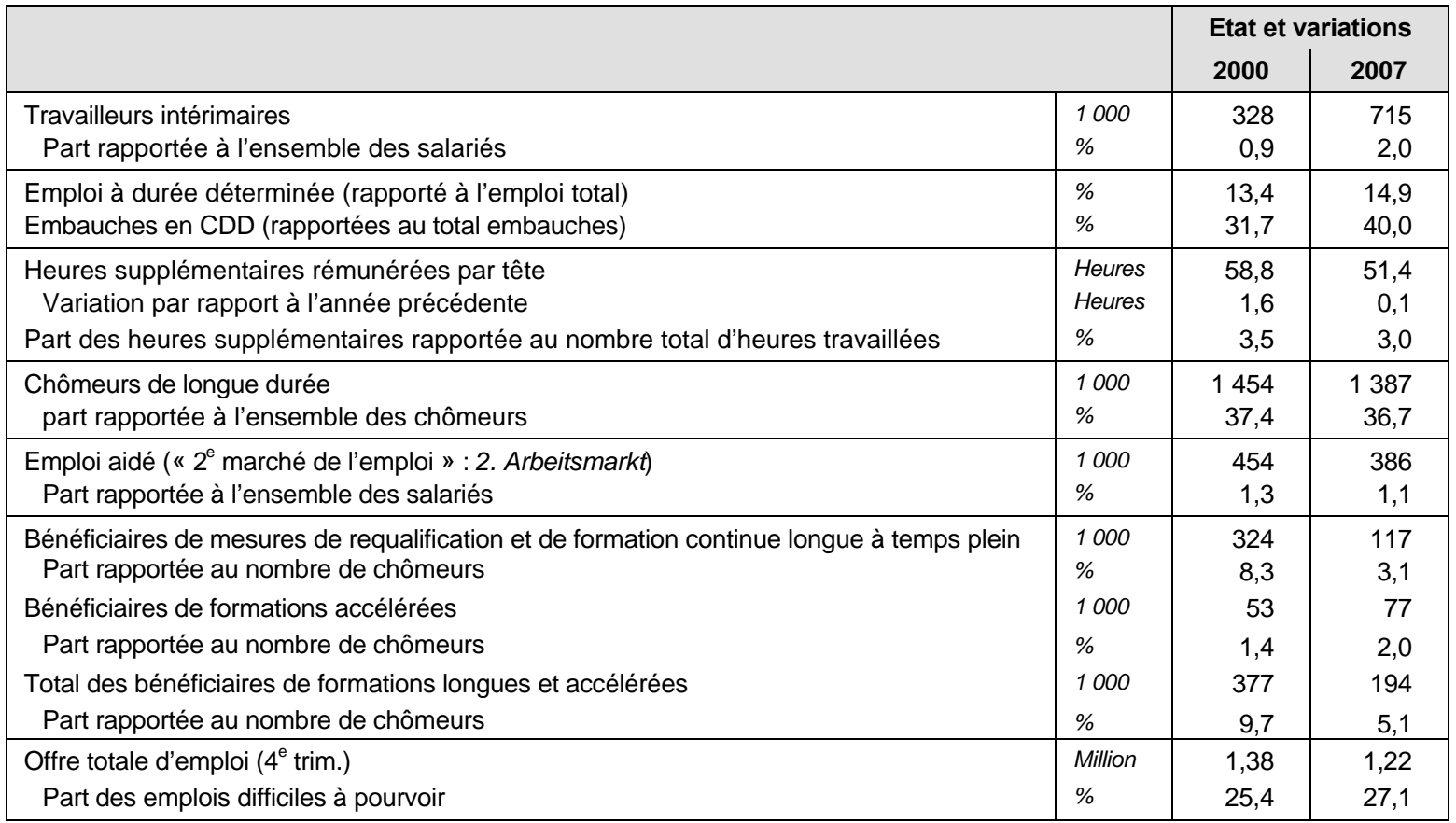

Sources des données : Destatis, Agence fédérale pour l'emploi, calculs de l'Institut für Arbeitsmarkt und Berufsforschung FG AZ). 
Dans la période 2006-2008, l'évolution revêt un caractère très différent, avec la création d'un nombre considérable d'emplois à temps plein $(550000)$. L'emploi à temps partiel connaissait pour sa part une hausse beaucoup moins forte (824 000) que précédemment et concernait surtout les temps partiels 'classiques', soumis à cotisations sociales $(710000)$, le nombre des 'petits boulots', exonérés, eux, des cotisations sociales, n'augmentant que de 113000 . L'évolution du marché de l'emploi a donc été dans l'ensemble plus positive au début du millénaire que lors de la période de croissance 1999-2001.

Durant les années 2006-2008, tous secteurs confondus, le nombre total des heures travaillées et rémunérées, ou volume d'heures travaillées, a connu une hausse beaucoup plus forte $(+3,7 \%)$ qu'en 1999-2001, où il n'avait crû que de $0,6 \%$. Autrement dit, considérée sous l'angle du volume d'heures travaillées, la croissance de ces trois dernières années a été beaucoup plus créatrice d'emplois que celle du tournant du siècle.

La croissance a visiblement incité les entreprises à augmenter le volume d'heures travaillées nécessaire à la production de biens et de services. Et elles ont largement créé des emplois à temps plein. En effet, la confiance des entreprises dans le caractère durable de la croissance était plus solide que durant la période 19992001. Elle était entretenue notamment par la modération salariale des années précédentes ainsi que par d'importants progrès en matière de flexibilisation du travail et les diverses réformes engagées au niveau du cadre des activités. Ainsi, les enquêtes menées auprès des entreprises ont révélé qu'il leur était plus facile de pourvoir des postes vacants depuis que le marché du travail avait fait l'objet de réformes. Car les chômeurs sont plus clairement incités depuis au retour à l'emploi et se montrent plus actifs aussi dans la recherche d'un emploi. Et dans le même temps, sous l'effet notamment de la levée des rigidités du marché de l'emploi, la progression du coût du travail est restée inférieure à celle de l'inflation.

Le travail temporaire a joué un rôle majeur pour le développement de l'emploi, surtout à temps plein, toutefois dans une proportion de plus en plus faible au cours de la période 2006-2008. Ainsi, au premier semestre 2006, l'augmentation des emplois soumis à cotisations sociales était due uniquement au recrutement massif par les sociétés d'intérim. Au second semestre, la tendance a commencé à s'inverser. A la fin de l'année 2006, on dénombrait 430000 emplois soumis à cotisations sociales de plus que l'année précédente, mais le travail intérimaire ne contribuait déjà plus qu'à un peu moins de $40 \%$ à cette augmentation. Au total, sur l'ensemble de la période 2006-2008, l'intérim a contribué à hauteur de $25 \%$ à la hausse globale de l'emploi, et même à hauteur de près de $60 \%$ à la création de postes à temps plein. Indéniablement, l'intérim joue aujourd'hui un rôle plus important sur le marché de l'emploi que par le passé : en moyenne annuelle, les sociétés de travail temporaire employaient plus de 700000 personnes en 2007, soit plus du double de leur effectif sept ans plus tôt (330 000 salariés). Si les intérimaires constituent désormais $2 \%$ des actifs occupés en Allemagne (contre $0,9 \%$ en 2000), cette part n'en demeure pas moins relativement faible en comparaison internationale.

En raison de l'assouplissement du régime des contrats à durée déterminée, les employeurs y ont plus largement recouru, une tendance qui coïncide en partie avec la hausse de l'emploi intérimaire. Depuis 2001 (Loi sur le travail à temps partiel et les contrats à durée déterminée : Gesetz über Teilzeitarbeit und befristete Arbeitsverträge, $T z B f G$ ), un CDD peut être reconduit, sans avis motivé, trois fois de suite pour une durée totale maximale de deux ans. Ainsi, par exemple, en 2007 , quelque $40 \%$ des salariés nouvellement embauchés se sont vu proposer un CDD (contre $32 \%$ seulement en 2000). La tendance à l'emploi à durée déterminée va croissant, les entreprises le prisant de plus en plus, semble-t-il, comme un outil de flexibilité. Néanmoins, la part des personnes sous contrat à durée déterminée rapportée à la totalité des actifs occupés, bien que stable, reste relativement faible (quelque $15 \%$ ). Cette différence importante entre les données rela-
2006-08 : création d'emplois à temps plein et d'emplois à temps partiels 'classiques'...

. et forte augmentation du volume d'heures travaillées...

...grâce à l'amélioration des conditions cadre

Forte contribution de l'intérim à la création d'emplois à temps plein

Le CDD s'impose comme outil de flexibilité 
Recul du nombre d'heures supplémentaires payées

Généralisation des comptes épargne-temps

Une inadéquation croissante entre offre et demande....

.. mais pas de manque généralisé de main-d'œuvre

Hausse du nombre d'actifs en 1999-2001... tives au flux et celles relatives au stock des emplois à durée déterminée renvoie à une double tendance, qui reste cependant délicate à traduire en chiffres avec précision : les CDD ne sont qu'en partie des emplois précaires et peu durables, puisqu'une partie d'entre eux se prolongent en contrats à durée indéterminée.

Elément clef de flexibilité pour les entreprises, les heures supplémentaires ont connu de profondes mutations ces dernières années. Lors des années de croissance 2006-2008, le nombre d'heures supplémentaires rémunérées a fortement chuté. Il est tombé en 2007 à 51 heures en moyenne par salarié, alors qu'il était encore de 59 en 2001. Et entre 2006 et 2008, il a baissé d'une heure. Le contraste est net avec la période 1999-2001, où on enregistrait au contraire une hausse de deux heures. De même, la part des heures supplémentaires rémunérées, qui représentait $3,5 \%$ du total du volume d'heures travaillées au tournant du millénaire, est tombée à $3 \%$ au cours des trois dernières années.

Le nombre d'heures supplémentaires rétribuées tend à diminuer depuis un certain temps déjà, du fait de la généralisation progressive de la flexibilisation du temps de travail. Aujourd'hui, quelque $40 \%$ des salariés travaillent sous le régime des comptes épargne-temps qui leur permet de compenser les phases temporaires de surcroît d'activité par des jours de congés susceptibles d'être pris en période d'activité plus réduite. Les soldes des comptes épargne-temps ont augmenté de 3,6 heures par salarié entre 2006 et 2008. En 1999-2001, la hausse n'était que de 2,5 heures.

Un nombre croissant d'heures supplémentaires payées qui, la plupart du temps, ouvrent droit à une rémunération compensatoire, a ainsi été remplacé par ces heures supplémentaires qui, fournies à titre transitoire lors des pics d'activité, ouvrent droit, dans le cadre des comptes épargne-temps, à une compensation sous forme de congé. Quant aux comptes épargne-temps établis sur le long terme (ils sont contractuels), qui permettent, en compensation des heures supplémentaires travaillées, d'accumuler un volume d'heures ouvrant droit à des formations continues longues, à des congés de longue durée pour assistance familiale ou au régime de retraite anticipée, ils ont certes gagné en importance, mais ils jouent toujours un rôle marginal. Au total, la flexibilisation des contrats de travail comme du temps de travail a contribué à réduire le coût du travail.

Durant les deux phases de croissance 1999-2001 et 2006-2008, le marché de l'emploi a cependant connu des tensions : il est devenu plus difficile qu'auparavant de pourvoir les postes vacants. Les diverses enquêtes menées auprès des entreprises confirment l'hypothèse que, sans les réformes du marché de l'emploi, les difficultés à pourvoir les postes vacants seraient nettement plus prononcées.

L'offre d'emploi globale, c'est-à-dire la somme des emplois vacants déclarés et non déclarés, a connu une hausse comparable durant les deux périodes considérées. Ainsi au quatrième trimestre 2000, on dénombrait 1,38 million d'emplois vacants contre 1,22 million au quatrième trimestre 2007. De la même manière, la répartition entre les différentes composantes de la demande, comme la part des postes à pourvoir immédiatement, à une date ultérieure ou encore les postes déclarés, a elle aussi peu varié.

Les emplois proposés sont restés vacants plus longtemps : la part des postes restés non occupés à l'issue d'une longue période de recherche de postulants a augmenté dans la même proportion, atteignant $27 \%$ en 2007 contre $25 \%$ en 2000. Par ailleurs, les entreprises évoquent un manque analogue, général, de main-d'œuvre limitant leur activité. Mais elles ne sont que $8 \%$ respectivement en 2000 et 2007 à constater un tel déficit ; il ne peut donc être question d'une tendance généralisée venant freiner durablement la croissance.

L'évolution de l'offre a été elle aussi très différente d'une période de croissance à l'autre, modifiant sensiblement la donne sur le marché de l'emploi.

En 1999-2001, bien que la population allemande en âge de travailler ait déjà commencé à diminuer, le potentiel d'actifs s'était considérablement renforcé, 
comptant 500000 personnes de plus. Les effets négatifs du vieillissement démographique ont été compensés par l'augmentation de la part des actifs mais aussi par l'arrivée d'immigrants sur le marché du travail.

Pendant la période 2006-2008 en revanche, le potentiel d'actifs a chuté après une longue période de croissance (-170 000 personnes), l'accroissement du nombre des actifs et l'immigration ne suffisant plus à combler les effets du recul lié à l'évolution démographique.

Si les variations du côté de l'offre ont donc pesé sur le marché de l'emploi durant la première période considérée, elles ont au contraire, durant la deuxième, contribué à lever les tensions.

\section{Changement de paradigme en matière de politique de l'emploi}

Dans les deux périodes, les mesures prises dans le cadre de la politique active pour l'emploi diffèrent également dans leurs orientations.

Durant les années 1999-2001, priorité était donnée aux mesures actives d'aide à l'emploi, qu'elles aient une visée générale de soutien au retour en emploi comme le programme de création d'emplois aidés dans les services d'intérêt général (Arbeitsbeschaffungsmaßnahmen : $A B M$ ) ou qu'elles soient dédiées au soutien à l'emploi dans les régions en restructuration, surtout dans les nouveaux Länder (Strukturanpassungsmaßnahmen : SAM). Ces programmes avaient pour objectif de réduire les tensions sur le marché de l'emploi en misant sur le développement d'un segment non marchand (le « $2^{\mathrm{e}}$ marché de l'emploi ») grâce à la création d'emplois temporaires dans le secteur public ou para-public, cofinancés par l'Agence fédérale pour l'emploi. S'ils ont joué un rôle non négligeable dans la hausse de l'activité durant cette période, ils n'ont cependant pas tenu leurs promesses en matière d'intégration, les personnes embauchées au titre de ces mesures se retrouvant généralement par la suite à nouveau sans emploi.

Dans la période 2006-2008 à l'inverse, ce " $2^{\mathrm{e}}$ marché de l'emploi » se résumait pour l'essentiel aux «jobs à un euro ", introduits dans le cadre des réformes Hartz et qui avaient quant à eux pour objectif d'inciter activement les chômeurs au retour à l'emploi. Leur approche était donc de préparer la réinsertion dans le marché du travail (secteur marchand ou « $1^{\text {er }}$ marché du travail »), contrairement aux mesures $A B M$ qui, dès lors, avaient été considérablement réduites. Or cette politique ne s'est pas traduite par une hausse générale de l'emploi, étant donné que l'emploi aidé s'est stabilisé au niveau atteint en 2005. Et, en 2007, il ne représentait plus que $1,1 \%$ de l'emploi total, contre 1,3\% en 2000 .

Outre les mesures destinées à la création d'emplois dans le secteur non marchand, la politique active pour l'emploi comprend traditionnellement des programmes de subvention à l'emploi dans le secteur marchand (subventionnement des cotisations sociales, part patronale ou salariale). Ces politiques ont connu une ampleur comparable au cours des deux périodes de croissance considérées. S'il est difficile de chiffrer avec précision l'impact net de ces mesures sur le marché de l'emploi, il n'en reste pas moins qu'elles accroissent sensiblement les perspectives de réintégration durable dans le « $1^{\text {er }}$ marché de l'emploi » pour leurs bénéficiaires. Toutefois, dans les périodes où la demande d'emploi s'intensifie, cette politique s'accompagne du risque non négligeable : celui de susciter des effets d'aubaine.

Le soutien à la formation professionnelle continue se présente lui aussi sous des jours différents au cours des deux périodes considérées. Ainsi, en 1999-2001, ce sont surtout des formations continues à temps plein et sur des périodes relativement longues qui sont proposées : en moyenne annuelle, quelque 320000 personnes en bénéficiaient en 2001 ; en 2007 , ce nombre est tombé à quelque 130 000. La durée des formations a en effet été fortement réduite afin d'accroître leur efficience en termes de qualification comme de coûts. Par ailleurs, on observe depuis quelque temps la multiplication des mesures de formation accélérée ...suivie d'un recul en 2006-2008

1999-2001 : multiplication des emplois aidés dans le segment non marchand

2006-2008:

la part de l'emploi aidé joue un rôle marginal

Emploi subventionné : effets positifs de réinsertion, mais aussi des effets d'aubaine

Réduction des mesures de qualification en dix ans 
La loi « Hartz IV » a modifié le cadre réglementaire, ...

... ce qui a réduit le sous-emploi destinées à inciter activement les chômeurs à retrouver un emploi. Et, rapportée au nombre total des sans emploi, la part des bénéficiaires d'une formation professionnelle continue longue a diminué de moitié entre les deux périodes.

Au total, les instruments de politique active pour l'emploi que sont les mesures de qualification et de création d'emplois dans le secteur non marchand ont eu un impact nettement moins positif sur le marché de l'emploi en 2006-2008 qu'en 1999-2001.

\section{Après 2005, nouvelle réglementation et baisse du chômage}

Les réformes introduites par la loi « Hartz IV » en 2005 ont profondément modifié l'environnement réglementaire du marché de l'emploi ; et elles ont permis de révéler une partie du sous-emploi larvé. Elles se sont en effet accompagnées d'un changement de paradigme : mettre fin à l'état de nécessité par l'insertion en emploi a désormais priorité sur le seul soutien pécuniaire à l'individu pour lui permettre d'assurer sa subsistance. L'objectif déclaré de la réforme est d'activer la responsabilité individuelle du demandeur d'emploi pour lui permettre d'assurer luimême sa subsistance grâce à son insertion, ce que résume la formule consacrée : « activation et participation » (Aktivierung und Teilhabe). La forte hausse du nombre de chômeurs au cours de l'année 2005 est dès lors à attribuer à tout un ensemble de changements : le nombre d'actifs occupés a reculé, l'offre d'emploi a augmenté, l'offre des mesures de création d'emplois aidés et de soutien à la formation continue longue a été réduite, et le chômage occulte a été partiellement intégré dans les statistiques du chômage.

Mais dans les trois années de croissance qui ont suivi, le chômage a baissé de près de 1,6 million de personnes, et la réserve latente de main-d'œuvre diminuait de 240000 personnes. Au total, le stock de sous-emploi global s'est réduit de 1,8 million de personnes.

A l'inverse, pendant la période de croissance 1999-2001, le recul du sous-emploi a été plus faible (900 000 personnes de moins seulement). Et il se répartissait de manière à peu près égale entre le chômage 'officiel' et le chômage caché' : au cours de ces trois années, le nombre des chômeurs a baissé de 430000 en données administratives, la réserve latente se réduisant de 450000 personnes.

Le fait que le sous-emploi ait connu une plus forte baisse dans les années 20062008 en comparaison avec 1999-2001 est dû aux changements décrits ci-dessus dans l'articulation entre offre et demande sur le marché de l'emploi. Quant aux différences constatées dans le recul respectivement du chômage recensé et du chômage caché, elles sont dues à un cadre réglementaire comme à des orientations en matière de politique de l'emploi foncièrement distinctes de celles de 1999-2001. D'un côté, la réserve latente stricto sensu a été, globalement, moins mobilisée par la dynamique de croissance conjoncturelle, étant donné qu'une grande partie des actifs concernés est comptabilisée depuis la réforme de 2005 dans les statistiques du chômage (données administratives). Et la partie restante correspondait moins à une demande de main-d'œuvre démultipliée par la croissance conjoncturelle. D'un autre côté, les « réformes Hartz » ont commencé à déployer leurs effets durant les années 2006-2008. La mise en pratique du nouveau paradigme de la politique active pour l'emploi, recentré sur les droits et les devoirs des bénéficiaires de la protection chômage (et résumés sous la formule «Soutenir et exiger » : "Fördern und Fordern » qui figure en tête du nouveau Chapitre II du Code social) a certainement eu pour conséquence l'incitation pour les chômeurs à se mettre en quête active d'un emploi et, pour d'autres chômeurs inscrits, à une tendance accrue de retour dans la réserve latente.

\section{Les effets de la réforme « Hartz IV ॥ restent partiellement à évaluer}

2006-2008 un tiers de la croissance dû à l'impact des réformes structurelles
La croissance de l'économie allemande au cours de ces dernières années a résulté essentiellement de l'effet conjugué d'une conjoncture mondiale porteuse, des réformes structurelles menées par les pouvoirs publics en matière de poli- 
tique économique et sociale et de la politique salariale modérée menée par les partenaires sociaux. Un tiers de la croissance du PIB est attribué à l'impact des réformes structurelles (Grömling et al., 2007). Reste à savoir quel rôle ont joué précisément dans ce contexte les réformes du marché du travail ; leur évaluation scientifique n'est pas encore achevée.

La réforme du Chapitre II du Code social allemand (Sozialgesetzbuch II : SGB II) qui instaure l'Arbeitslosengeld II constitue la dernière étape des réformes du marché de l'emploi menées de 2003 à 2005. L'objectif déclaré de cette nouvelle prestation est de "renforcer la responsabilité individuelle des personnes nécessiteuses à capacité de travail entière »(SGB II, § 1,1) afin de leur permettre la réinsertion, et donc de prendre à nouveau part à la vie active.

On attendait beaucoup de cette réforme, qui suscitait par ailleurs de grandes craintes. En effet, tandis qu'on espérait d'un côté que seraient données de nouvelles impulsions pour le marché de l'emploi, allant de pair avec la compression du chômage de longue durée, on redoutait de l'autre une diffusion de l'emploi précaire, s'accompagnant d'un risque accru de paupérisation.

Trois ans après, bien qu'il ne soit pas encore possible de dégager une analyse exhaustive de l'impact de cette réforme sur l'économie allemande, certains éléments se détachent cependant.

La réduction du montant comme de la durée de versement des prestations de transfert ( $A L G$ I et $A L G$ I) ont indéniablement constitué une incitation à la recherche active d'un emploi (Arntz et al., 2007). Les entreprises affirment de plus en plus souvent qu'elles parviennent à embaucher plus rapidement des candidats extérieurs à l'entreprise, même pour des postes difficiles à pourvoir ; quant aux actifs, ils acceptent plus facilement de faire des concessions - qu'ils soient demandeurs d'emploi ou qu'ils fassent partie des «insiders ", les effectifs en CDI d'une entreprise (Kettner/Rebien, 2007a). Cette flexibilisation semble toutefois avoir pour corollaire une tendance croissante à l'exclusion des actifs les moins qualifiés.

Presque toutes les mesures de soutien actif à l'emploi que régit le Chapitre III du Code social (SGB III) sont susceptibles de s'appliquer également dans le cadre de la nouvelle politique sociale du Chapitre II (SGB II) qui porte sur le minimum garanti pour les demandeurs d'emploi. De ce fait, les études d'évaluation des instruments de la politique active pour l'emploi menées jusqu'à présent se sont concentrées essentiellement sur la question de savoir dans quelle mesure ceuxci se sont traduits par une insertion et une participation sur le marché de l'emploi (voir Koch/Kupka/Steinke, p. 268).

Les conclusions sont proches dans les deux cas : les mesures qui sont liées à une intégration directe dans l'entreprise sont toutes de loin les plus efficientes, qu'il s'agisse des perspectives d'activité sur le long terme ou de la fin de l'état de nécessité justifiant une aide. Les mesures de (re)qualification, de formation longue ou accélérée, de même que le placement par l'intermédiaire de tiers contribuent à accroître les perspectives de retour en activité. Enfin, il convient de relever que même dans la catégorie des personnes difficiles à placer, visée par les dispositions du SGB II, on observe une tendance à l'intégration en emploi contrairement au doute qui prévalait lors de l'adoption de la réforme. Autrement dit, les instruments de la politique active pour l'emploi sont susceptibles de déployer leurs effets y compris dans cette catégorie d'actifs.

Un impact négatif s'observe en revanche en ce qui concerne les jobs à un euro. Ces derniers semblent non seulement évincer les emplois dits 'réguliers', mais aussi ne mener que rarement leurs bénéficiaires à un emploi pérenne (Kettner/ Rebien, 2007b).

On assiste ici à une expansion du secteur des bas salaires, certes voulue par le législateur, mais qui se révèle problématique car elle nécessite l'adoption de mesures d'accompagnement sous la forme d'une politique salariale incitative. Car
Décrue du chômage de longue durée avec précarisation?

Un marché de l'emploi plus fluide, au détriment des moins qualifiés ?

Les mesures de la politique pour l'emploi bénéficient à toutes les catégories d'actifs

L'extension du secteur des bas salaires requiert des correctifs 
Priorité absolue à la qualification

En tout état de cause une adéquation accrue entre offre et demande si le secteur des bas salaires permet de créer ou de préserver des emplois pour les personnes peu qualifiées et à faible productivité, il n'offre pas à ces dernières des revenus suffisants pour couvrir leurs besoins. Seules des prestations de transfert permettent alors de contenir le risque de pauvreté sans nuire à l'incitation à l'activité.

Enfin, il serait nécessaire de développer des mesures de politique sociale pour les personnes impossibles à insérer en emploi. On pourrait ainsi imaginer la création d'un « $3^{\mathrm{e}}$ marché de l'emploi », secteur non marchand offrant un éventail de mesures d'aide et d'emplois subventionnés sur mesure car destiné aux besoins spécifiques des personnes non intégrables dans les deux autres marchés de l'emploi.

Mais plus généralement, priorité absolue doit être accordée à l'ensemble des mesures de formation professionnelle initiale et continue comme de requalification en prévision aussi du vieillissement démographique qui risque, à long terme, de se traduire par une pénurie de l'offre d'emplois, essentiellement d'emplois qualifiés.

Les effets de la réforme n'ont pas encore été suffisamment étudiés. II est trop tôt pour en mesurer l'impact, sachant par ailleurs que de nombreuses données ne sont accessibles qu'avec un certain décalage dans le temps (Koch/Kupka/ Steinke, 2008). On constate cependant un nombre croissant d'éléments attestant du recul du chômage structurel et du chômage de longue durée ainsi que de l'efficience accrue de la corrélation entre offre et demande sur le marché de l'emploi (Bach et al., 2009).

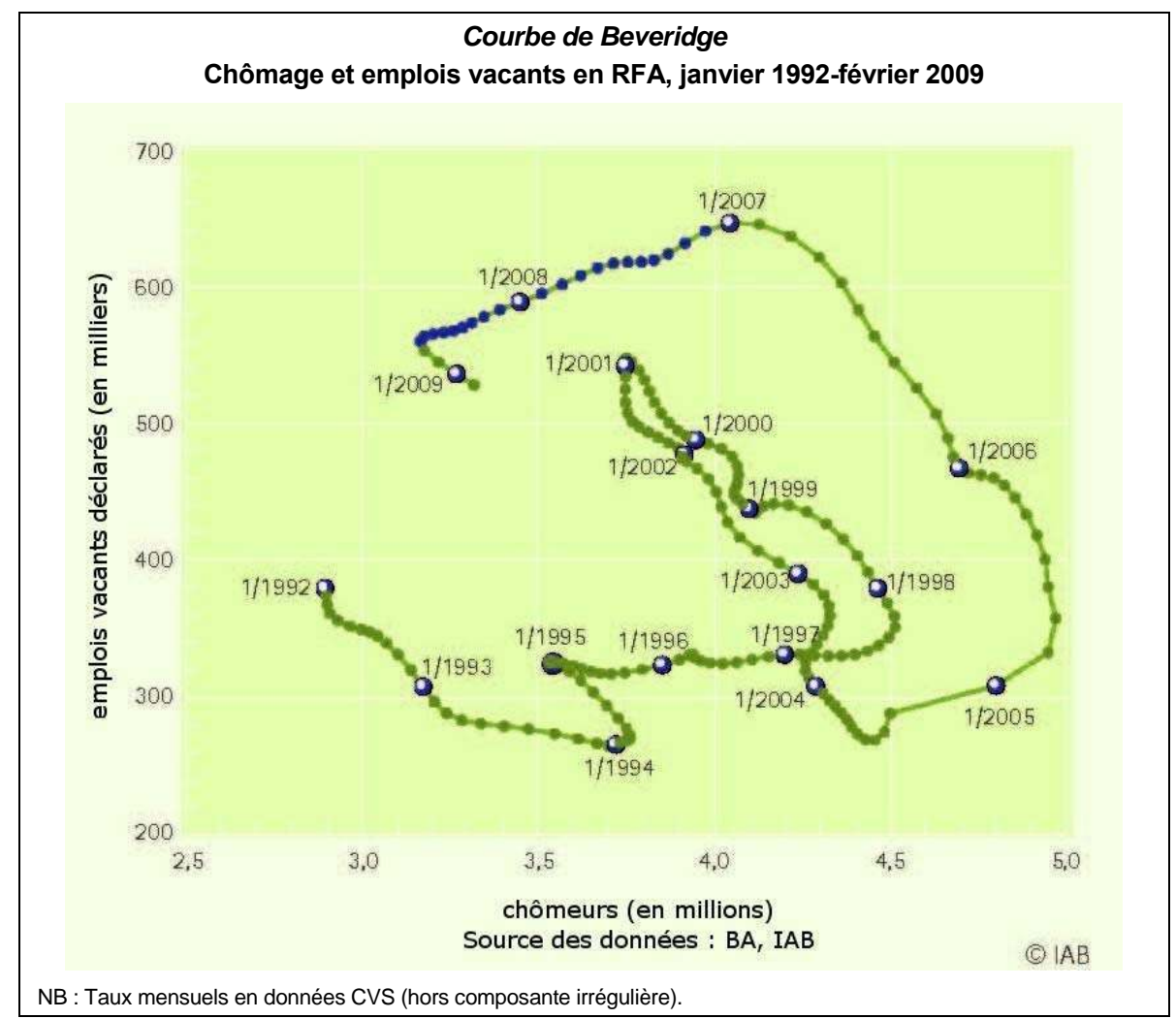

Ainsi la courbe de Beveridge, indiquant la relation entre le taux de chômage et le taux de postes vacants, s'est modifiée lors de la dernière période de croissance économique : pour le même nombre de postes vacants, on compte désormais un nombre moins important de chômeurs. La courbe de Phillips présente des caractéristiques similaires, le fort recul du chômage s'accompagnant d'une évolution relativement stable des prix. 


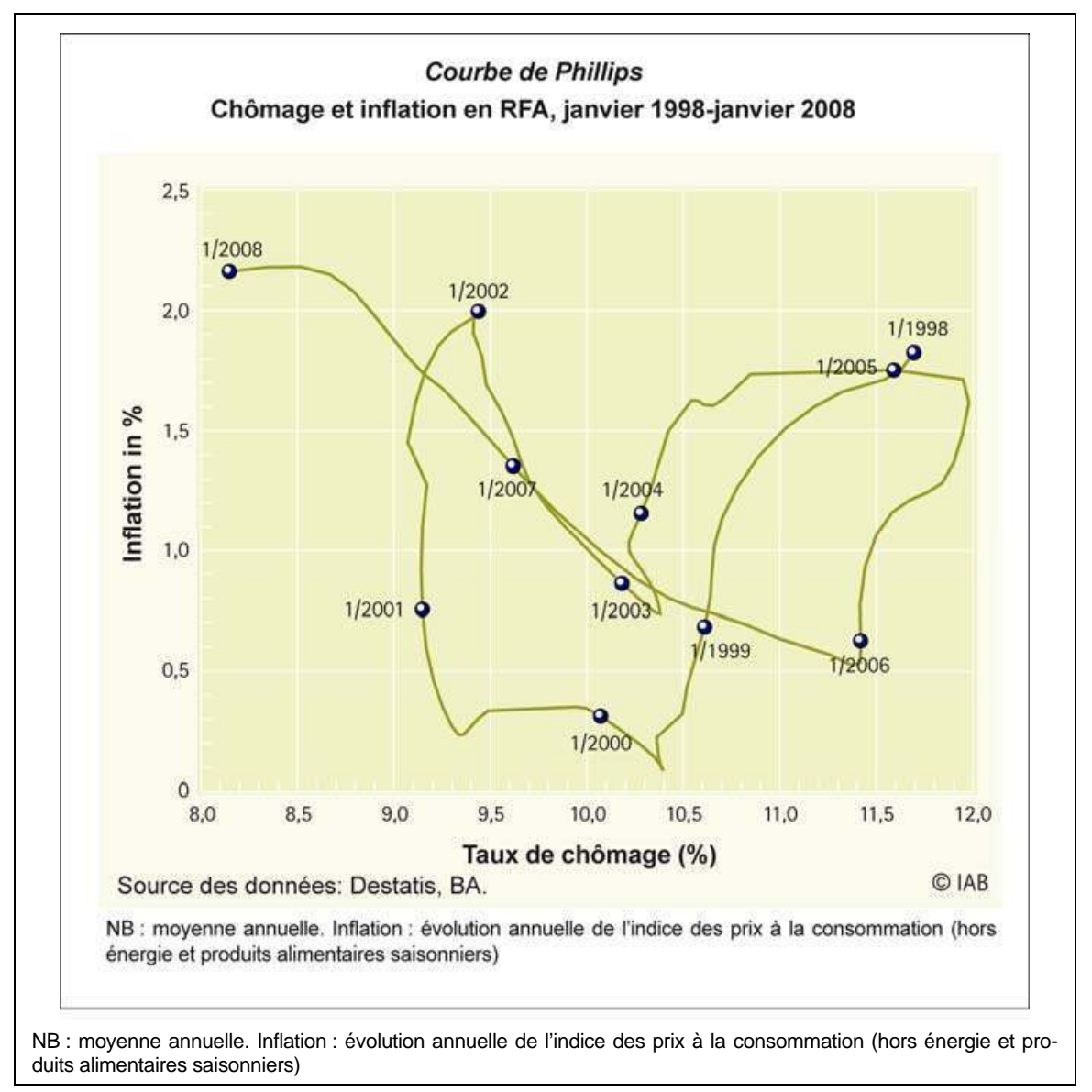

LES REPERCUSSIONS MASSIVES DE LA CRISE FINANCIERE MONDIALE sur l'économie réelle et le marché de l'emploi placent les acteurs politiques devant de nouveaux et considérables défis. Si l'on croyait encore, voici un an seulement, l'Allemagne sur la voie du retour au plein emploi, cet objectif s'est depuis déplacé vers un futur hypothétique. Et même si le creux de la récession est atteint au deuxième semestre et que la conjoncture donne des signes de reprise, rien ne laisse présager d'une prompte embellie sur le marché de l'emploi. L'expérience a montré que celui-ci réagit toujours avec un temps de retard, notamment parce que les entreprises cherchent en priorité à épuiser leurs marges de manœuvre en matière de productivité comme de temps de travail avant de procéder à des embauches massives. Du fait des réformes structurelles de ces dernières années, des programmes de soutien à la conjoncture adoptés en réaction à la crise, mais aussi d'une évolution démographique qui contracte l'offre de main-d'oeuvre, le marché de l'emploi semble toutefois mieux préparé qu'autrefois à affronter la crise.

Traduction : I. Bourgeois et S. Hazouard

\section{Indications bibliographiques}

- Arntz M., Clauss M., Kraus M., Schnabel R., Spermann A., Wiemers J., « Arbeitsangebotseffekte und Verteilungswirkungen der Hartz-IV-Reform ", IAB-Forschungsbericht Nr. 10/2007, IAB, Nuremberg, 2007

- Bach H.-U., Gartner H., Hummel M., Klinger S., Rothe T., Spitznagel E., Zika G., « Projektion 2009 - Arbeitsmarkt im Sog der Rezession », IAB-Kurzbericht Nr. 6/2009, Nuremberg, 2009

- BACH H.-U., SpItZnAGel E., "Aufschwung am Arbeitsmarkt : Die Entwicklungsmuster wandeln sich », IAB-Forum, vol. 2, 2007

- BouRgeols I. (dir), Le modèle social allemand en mutation, Travaux et Documents du CIRAC, Cergy-Pontoise, 2005 
- Grömling M., Plünnecke A., Scharnagel B., « Was trägt die Politik zum Aufschwung bei? », IW-Trends Nr.4/2007, Cologne, 2007

- Kettner A., Rebien M., « Hartz-IV-Reform : Impulse für den Arbeitsmarkt », IAB-Kurzbericht Nr. 19/2007, Nuremberg, 2007(a)

- Kettner A., Rebien M., « Soziale Arbeitsgelegenheiten : Einsatz und Wirkungsweise aus betrieblicher und arbeitsmarktpolitischer Perspektive », IAB-Forschungsbericht Nr. 2/2007, Nuremberg, 2007(b)

- Косн S., Kupka P., Steinke J., « Aktivierung, Erwerbstätigkeit und Teilhabe. Vier Jahre Grundsicherung für Arbeitsuchende », IAB-Bibliothek Bd. 315, Nuremberg, 2008 